$\xi=-1$

\title{
Developing A Strategy Map Based on Sustainability Balanced Scorecard Framework for Manufacturing Industry in Indonesia
}

\author{
Rahmat Nurcahyo *, Saripuji Pustiwari, Djoko Sihono Gabriel \\ Department of Industrial Engineering Universitas Indonesia Depok, 16424, Indonesia \\ *Corresponding author E-mail: rahmat@eng.ui.ac.id
}

\begin{abstract}
This study proposes a structural evaluation to link key performance indicator into a strategy map based on sustainability balanced scorecard framework for manufacturing industry in Indonesia. With four perspectives (finance, stakeholder, internal business process and learning and growth) on Sustainability Balanced Scorecard, the evaluation of relationship between perspectives and indicators of manufacturing industry are synthesized from relevant literature and experts. The Decision Making Trial and Evaluation Laboratory (DEMATEL) method employed to identify critical central and influential factors, to determine the causal relationship and finally to develop a visual strategy map to improve corporate sustainability. The three most important indicators are sustainability award, certification of environmental and social standards and resource productivity. The result can help prioritizing the performance indicators and show which areas that need improvement most.
\end{abstract}

Keywords: DEMATEL, Indicator, Strategy map, Sustainability balanced scorecard.

\section{Introduction}

Companies around the world are currently under pressure from both customers, regulators and stakeholders to improve environmental and social responsibility. For example, investors are no longer looking only at financial statements, but also look for environmental and social management that companies have adopted [1]. As a result, improving sustainability, reducing the negative environmental and social impacts of industrial are no longer just nice-to-have, but imperative and could enhance competitive advantage [2].

A concept called Sustainability Balanced Scorecard was developed by Reference [3]. This concept integrate sustainability issues into a Balanced Scorecard perspectives. Compared to representing strategy as a group of single indicator, SBSC connects cause and effect factors. The current research focuses on framework development, while there is no research on strategic map design showing the relationship between SBSC indicators. The strategy map shows the causal relationship between indicators reflecting the dynamic changes and indicates how the organization creates values [4]. This strategy map could be used by decision makers to improve the corporate sustainability [5].

Designing a strategy map is visualization of causal relationship of a company strategy. Research on SBSC strategy map could be used to improve the ability to explain, evaluate, manage and implement desired strategies. The strategy map itself will provide a visual framework and a concise description of an organization's strategy, then it can convert the intangible assets into tangible results.

This study will be tested in Indonesia's manufacturing industry. According to Reference [6], Indonesia has entered top 10 manufacturing in the world. In addition, the manufacturing industry in Indonesia contributes almost $25 \%$ of the national GDP. Reference
[7] shows that Indonesia ranked 19th in the Global Manufacturing Competitiveness Index and is projected to rise to 15 th by 2020 .

\section{Theoretical background}

Sustainability has evolved into an issue that draws a lot of attention from various parties including researchers and academics [8]. The word 'sustainability' was first introduces in the context of sustainable development defined by Reference [9] as: "Sustainable development is a development that meets the needs of the present without compromising the ability of future generations to meets their own needs".

Reference [10] states the concept of sustainability is often represented as a combination of three dimensions of social, economic and environmental. The SBSC concept is derived from the conventional balanced scorecard by integrating environmental and social issues as an important pillar of sustainability. Reference [12] states that sustainability management with the help of balanced scorecard will be able to integrate the three pillars of sustainability into a single, comprehensive management tool. Therefore, SBSC not only detect social or environmental objectives but also increase the added value transparency that comes from social or ecological aspects and prepares the implementation process of the strategy [13].

Reference [14] states there are three principles that connect an organization's balanced scorecard with its strategy, namely (1) causal relationship, (2) performance drivers and (3) linkage to financial goals. Strategies are mad from hypotheses of cause and effect. The strategy map describes a causal relationship in a sequence. The causal chain is connected to all indicators through four balanced scorecard perspectives reflecting dynamic changes and indicating how the organization creates value [15]. 
Strategy map is the most important procedure in establishment of balanced scorecard, because the strategy map can be viewed as a hypothesis of the relationship between objectives measured by key performance indicator (KPI). So, designing a strategy map with a clear causal relationship can lead to the establishment of a strategic path within the organization [16]. However, the failure of balanced scorecard implementation often occurs due to errors in strategy mapping [17].

DEMATEL stands for "Decision Making Trial and Evaluation Laboratory", the main purpose of DEMATEL is to study and solve complex and interrelated problems [18] with the basic concept of measuring the level of influence of factor with other factors [19]. The DEMATEL model can improve understanding of a specific problem, which is interconnected with one another and identify solutions to a problem in a structured way [20] using the knowledge possessed by experts in the field [21].

\section{Research method}

Indicators used in this study taken from research conducted by Reference [22] and additional indicators from Industri Hijau (the Green Industry Awards) from the Indonesia Ministry of Industry [23]. The Industri Hijau is an annual program aimed at motivating industry to implement sustainability issues. Table I give the final list of 20 indicators.

Table: 1 List of SBSC Indicators

\begin{tabular}{|c|c|c|}
\hline \multicolumn{2}{|r|}{ Learning \& Growth (LG) } & Reference \\
\hline LG2 & Employee training & Ref. [22] \\
\hline LG3 & $\begin{array}{c}\text { Sustainable consciousness of top manage- } \\
\text { ment }\end{array}$ & Ref. [22] \\
\hline LG4 & Environmental information systems & Ref. [22] \\
\hline LG5 & Innovation of green technology & Ref. [23] \\
\hline \multicolumn{2}{|r|}{ Internal Business Proccess (IBP) } & \\
\hline IBP1 & Assessment of product life cycle & Ref. [22] \\
\hline IBP3 & Production efficiency & Ref. [23] \\
\hline IBP4 & Employee accidents & Ref. [22] \\
\hline IBP5 & Waste treatment facilities & Ref. [23] \\
\hline IBP6 & Material efficiency & Ref. [23] \\
\hline IBP7 & $\begin{array}{c}\text { Certification of environmental and social } \\
\text { standards }\end{array}$ & Ref. [22] \\
\hline \multicolumn{2}{|r|}{ Stakeholder (SH) } & \\
\hline $\mathrm{SH} 2$ & $\begin{array}{l}\text { Fulfillment of environmental quality stand- } \\
\text { ards }\end{array}$ & Ref. [23] \\
\hline SH3 & Customer satisfaction & Ref. [22] \\
\hline SH6 & Health and safety of employee & $\begin{array}{l}\text { Ref. [22]; Ref. } \\
\text { [23] }\end{array}$ \\
\hline $\mathrm{SH} 10$ & Sustainability awards & $\begin{array}{l}\text { Ref. [22]; Ref. } \\
\text { [23] }\end{array}$ \\
\hline SH11 & CSR program & $\begin{array}{c}\text { Ref. [22]; Ref. } \\
\text { [23] }\end{array}$ \\
\hline $\mathrm{SH} 13$ & Standard ethic for stakeholder & Ref. [22] \\
\hline \multicolumn{2}{|r|}{ Financial $(\mathbf{F})$} & \\
\hline $\mathrm{F} 1$ & Green image & Ref. [22] \\
\hline $\mathrm{F} 3$ & Operational cost & Ref. [22] \\
\hline $\mathrm{F} 4$ & Profit & Ref. [22] \\
\hline F5 & Resource productivity & Ref. [22] \\
\hline
\end{tabular}

The questionnare aims to examine the relationship between indicators and SBSC perspectives with DEMATEL method to design the strategy map. Respondents were asked to give value with scale of $0-4$, where $(0)$ indicated no influence and (4) indicated very strong influence.The participants in this study included managers that have more than seven years' experience in manufacturing industry in Indonesia as many as six respondents. Those selected respondents were managers that handling sustainability issues in companies. The sample selection for any empirical study is an important issue which determine the reliability of the results. Reference [24] divides DEMATEL into four main steps:

Step 1: Building a direct relationship matrix (Z). This value obtained from calculating the average score from questionnaire using (1).
$Z=\left\lfloor Z_{i j}\right\rfloor_{n x n}$

Step 2: Normalizing the direct relationship matrix (X). This value can be obtained through (2)-(3).

$X=s . Z$

$s=\frac{1}{\sum_{j=1}^{n} z_{i j}}, i, j=1,2, \ldots, n$

Step 3: Attaning total relationship matrix $(\mathrm{T})$. This value can be acquired by using (4).

$T=X(1-X)^{-1}$

Step 4: Producing causal diagram. The sum of rows and the sum of columns are separately denoted as vector D and R through (5)(7).

$T=\left\lfloor T_{i j}\right\rfloor_{n x n}$

$D=\left[\sum_{j-1}^{n} T_{i j}\right]_{n x 1}=\left[T_{i}\right]_{n x 1}$

$R=\left[\sum_{j-1}^{n} T_{i j}\right]_{1 x n}=\left[T_{j}\right]_{1 \times n}$

Table II and III give the direct relationship matrix (Z) and total relationship matrix $(T)$ of four perspective. While Table IV and Table $\mathrm{V}$ give direct relationship matrix $(\mathrm{Z})$ and the total relations matrix (T) for 20 indicators.

Table: 2 Direct relationship matrix for SBSC perspectives

\begin{tabular}{|c|c|c|c|c|}
\hline $\mathbf{Z}$ & LG & IBP & SH & F \\
\hline LG & 0 & 2.354 & 2.444 & 1.917 \\
\hline IBP & 1.528 & 0 & 1.977 & 2.389 \\
\hline SH & 1.674 & 1.468 & 0 & 2.340 \\
\hline F & 1.760 & 1.938 & 1.556 & 0 \\
\hline
\end{tabular}

Table: 3 Total relationship matrix for SBSC perspectives

\begin{tabular}{|c|c|c|c|c|}
\hline T & LG & IBP & SH & F \\
\hline LG & 0.112 & 0.285 & 0.295 & 0.278 \\
\hline IBP & 0.208 & 0.112 & 0.250 & 0.289 \\
\hline SH & 0.212 & 0.211 & 0.108 & 0.278 \\
\hline F & 0.213 & 0.237 & 0.216 & 0.117 \\
\hline
\end{tabular}

Table: 4 Direct relationship matrix for SBSC Indicator

\begin{tabular}{|c|c|c|c|c|c|c|c|c|c|c|c|c|c|c|c|c|c|c|c|c|}
\hline $\mathbf{Z}$ & $\begin{array}{l}\mathbf{L} \\
\mathbf{G} \\
\mathbf{2}\end{array}$ & $\begin{array}{l}\mathbf{L} \\
\mathbf{G} \\
\mathbf{3}\end{array}$ & $\begin{array}{l}\mathbf{L} \\
\mathbf{G} \\
\mathbf{4}\end{array}$ & $\begin{array}{l}\mathbf{L} \\
\mathbf{G} \\
\mathbf{5}\end{array}$ & $\begin{array}{l}\mathbf{I} \\
\mathbf{B} \\
\mathbf{P} \\
\mathbf{1}\end{array}$ & $\begin{array}{l}\mathbf{I} \\
\mathbf{B} \\
\mathbf{P} \\
\mathbf{3}\end{array}$ & $\begin{array}{l}\text { I } \\
\text { B } \\
\text { P } \\
4\end{array}$ & $\begin{array}{l}\mathbf{I} \\
\mathbf{B} \\
\mathbf{P} \\
\mathbf{5}\end{array}$ & $\begin{array}{l}\mathbf{I} \\
\mathbf{B} \\
\mathbf{P} \\
\mathbf{6}\end{array}$ & $\begin{array}{l}\text { I } \\
\text { B } \\
7\end{array}$ & $\begin{array}{l}\mathbf{S} \\
\mathbf{H} \\
\mathbf{2}\end{array}$ & $\begin{array}{l}\mathbf{S} \\
\mathbf{H} \\
\mathbf{3}\end{array}$ & $\begin{array}{l}\mathbf{S} \\
\mathbf{H} \\
\mathbf{6}\end{array}$ & \begin{tabular}{l|l}
$\mathbf{S}$ \\
$\mathbf{H}$ \\
$\mathbf{1}$ \\
$\mathbf{0}$
\end{tabular} & \begin{tabular}{l|}
$\mathbf{S}$ \\
$\mathbf{H}$ \\
$\mathbf{1}$ \\
$\mathbf{1}$
\end{tabular} & \begin{tabular}{l|l}
$\mathbf{S}$ \\
$\mathbf{H}$ \\
$\mathbf{1}$ \\
$\mathbf{3}$
\end{tabular} & $\begin{array}{l}\mathbf{F} \\
\mathbf{1}\end{array}$ & $\begin{array}{l}\mathbf{F} \\
\mathbf{3}\end{array}$ & $\begin{array}{l}\mathbf{F} \\
\mathbf{4}\end{array}$ & $\begin{array}{l}\mathbf{F} \\
\mathbf{5}\end{array}$ \\
\hline & 0 & 0 & 1 & 1 & 1 & 3 & 4 & 1 & 2 & 3 & 2 & 1 & 3 & 3 & 2 & 2 & 0 & 0 & 1 & 3 \\
\hline $\mathbf{G}$ & 0 & 6 & 5 & 6 & 6 & 3 & 0 & 3 & 3 & 3 & 3 & 5 & 6 & 3 & 3 & 3 & 3 & 6 & 0 & 6 \\
\hline 2 & 0 & 7 & 0 & 7 & 7 & 3 & 0 & 3 & 3 & 3 & 3 & 0 & 7 & 3 & 3 & 3 & 3 & 7 & 0 & 7 \\
\hline $\begin{array}{l}\mathbf{L} \\
\mathbf{G}\end{array}$ & $\begin{array}{l}2 \\
.\end{array}$ & 0 & 2 & 1 & 2 & 2 & 1 & 2 & 3 & 3 & 3 & 3 & 2 & 3 & 2 & 3 & 3 & 2 & 0 & 2 \\
\hline 3 & $\begin{array}{l}6 \\
7\end{array}$ & $\begin{array}{l}0 \\
0\end{array}$ & $\begin{array}{l}5 \\
0\end{array}$ & $\begin{array}{l}6 \\
7\end{array}$ & $\begin{array}{l}8 \\
3\end{array}$ & $\begin{array}{l}6 \\
7\end{array}$ & $\begin{array}{l}6 \\
7\end{array}$ & $\begin{array}{l}6 \\
7\end{array}$ & $\begin{array}{l}3 \\
3\end{array}$ & $\begin{array}{l}6 \\
7\end{array}$ & $\begin{array}{l}3 \\
3\end{array}$ & $\begin{array}{l}3 \\
3\end{array}$ & $\begin{array}{l}6 \\
7\end{array}$ & $\begin{array}{l}6 \\
7\end{array}$ & $\begin{array}{l}6 \\
7\end{array}$ & $\begin{array}{l}0 \\
0\end{array}$ & $\begin{array}{l}8 \\
3\end{array}$ & $\begin{array}{l}0 \\
0\end{array}$ & $\begin{array}{l}8 \\
3\end{array}$ & $\begin{array}{l}6 \\
7\end{array}$ \\
\hline $\mathbf{L}$ & 2 & 0 & 0 & 2 & 1 & 1 & 0 & 2 & 1 & 3 & 3 & 0 & 1 & 3 & 2 & 2 & 3 & 1 & 0 & 1 \\
\hline $\mathbf{G}$ & 3 & 3 & 0 & 5 & 6 & 1 & 3 & 6 & 1 & 1 & 8 & 3 & 1 & 3 & 3 & 1 & 6 & 3 & 8 & 3 \\
\hline 4 & 3 & 3 & 0 & 0 & 7 & 7 & 3 & 7 & 7 & 7 & 3 & 3 & 7 & 3 & 3 & 7 & 7 & 3 & 3 & 3 \\
\hline $\mathbf{L}$ & 1 & 0 & 2 & 0 & 3 & 1 & 0 & 3 & 1 & 3 & 3 & 0 & 0 & 3 & 2 & 1 & 3 & 1 & 1 & 1 \\
\hline $\mathbf{G}$ & 1 & 1 & 3 & 0 & 1 & 3 & 3 & 5 & 3 & 8 & 8 & 1 & 0 & 8 & 1 & 3 & 8 & 6 & 6 & 3 \\
\hline & 7 & 7 & 3 & 0 & 7 & 3 & 3 & 0 & 3 & 3 & 3 & 7 & 0 & 3 & 7 & 3 & 3 & 7 & 7 & 3 \\
\hline $\mathbf{I}$ & 2 & 2 & 1 & 1 & 0 & 2 & 0 & 2 & 3 & 3 & 2 & 1 & 0 & 3 & 1 & 1 & 0 & 2 & 2 & 1 \\
\hline $\mathbf{B}$ & . & & . & $\cdot$ & & $\cdot$ & . & & . & & & . & & & & & . & & . & . \\
\hline $\mathbf{P}$ & 1 & 3 & 3 & 1 & 0 & 5 & 5 & 3 & 3 & 5 & 6 & 3 & 5 & 5 & 8 & 5 & 6 & 3 & 1 & 1 \\
\hline 1 & 7 & 3 & 3 & 7 & 0 & 0 & 0 & 3 & 3 & 0 & 7 & 3 & 0 & 0 & 3 & 0 & 7 & 3 & 7 & 7 \\
\hline $\mathbf{I}$ & 2 & 2 & 0 & 0 & 3 & 0 & 1 & 1 & 3 & 1 & 1 & 2 & 2 & 3 & \begin{tabular}{l|l|}
0 \\
\end{tabular} & 0 & 0 & 3 & 4 & 3 \\
\hline $\mathbf{B}$ & . & & & & & & & & & & & & & & & & & & & \\
\hline $\mathbf{P}$ & 1 & 3 & 3 & 3 & 1 & 0 & 3 & 1 & 6 & 3 & 8 & 1 & 6 & 6 & 3 & 5 & 8 & 8 & 0 & 6 \\
\hline 3 & 7 & 3 & 3 & 3 & 7 & 0 & 3 & 7 & 7 & 3 & 3 & 7 & 7 & 7 & 3 & 0 & 3 & 3 & 0 & 7 \\
\hline $\mathbf{I}$ & 3 & 1 & 0 & 0 & 0 & 2 & 0 & 0 & 0 & 1 & 0 & 1 & 4 & 2 & 0 & 0 & 1 & 1 & 1 & 3 \\
\hline B & . & & . & & & & & & & & & & & & & & & & & \\
\hline D & 6 & 3 & 5 & 3 & 3 & 3 & 0 & 0 & 3 & 8 & 1 & 1 & 0 & 5 & 1 & 5 & 5 & 5 & 5 & 5 \\
\hline
\end{tabular}


\begin{tabular}{|l|l|l|l|l|l|l|l|l|l|l|l|l|l|l|l|l|l|l|l|l|}
\hline 4 & 7 & 3 & 0 & 3 & 3 & 3 & 0 & 0 & 3 & 3 & 7 & 7 & 0 & 0 & 7 & 0 & 0 & 0 & 0 & 0 \\
\hline
\end{tabular} \begin{tabular}{|l|lllllllllllll|llll|l|l|l|l|l|l|}
$\mathbf{4}$ & 7 & 3 & 0 & 3 & 3 & 3 & 0 & 0 & 3 & 3 & 7 & 7 & 0 & 0 & 7 & 0 & 0 & 0 & 0 & 0 \\
\hline $\mathbf{I}$ & 1 & 0 & 2 & 1 & 1 & 1 & 0 & 0 & 0 & 3 & 3 & 0 & 0 & 3 & 2 & 2 & 3 & 1 & 1 & 1 \\
\hline
\end{tabular}

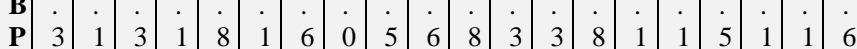
\begin{tabular}{llllllllllllllllllllll}
5 & 3 & 7 & 3 & 7 & 3 & 7 & 7 & 0 & 0 & 7 & 3 & 3 & 3 & 3 & 7 & 7 & 0 & 7 & 7 & 7 \\
\hline
\end{tabular} I $2 \begin{array}{lllllllllllllllllllllll} & 2 & 0 & 0 & 3 & 3 & 0 & 0 & 0 & 1 & 1 & 1 & 0 & 3 & 1 & 0 & 3 & 4 & 3 & 3\end{array}$ B

$\begin{array}{lllllllllllllllllllll}\mathbf{P} & 1 & 1 & 0 & 3 & 3 & 8 & 0 & 3 & 0 & 6 & 3 & 3 & 0 & 3 & 3 & 1 & 1 & 0 & 8 & 3\end{array}$ \begin{tabular}{lllllllllllllllllllllllll}
6 & 7 & 7 & 0 & 3 & 3 & 3 & 0 & 3 & 0 & 7 & 3 & 3 & 0 & 3 & 3 & 7 & 7 & 0 & 3 & 3 \\
\hline
\end{tabular}

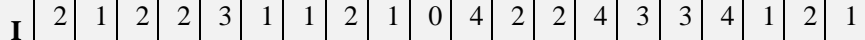
$\begin{array}{lllllllllllllllllllll}\mathbf{B} & 5 & 6 & 5 & 3 & 3 & 3 & 1 & 5 & 6 & 0 & 0 & 3 & 3 & 0 & 6 & 6 & 0 & 5 & 1 & 1\end{array}$ \begin{tabular}{lllllllllllllllllllll}
7 & 0 & 7 & 0 & 3 & 3 & 3 & 7 & 0 & 7 & 0 & 0 & 3 & 3 & 0 & 7 & 7 & 0 & 0 & 7 & 7 \\
\hline
\end{tabular}

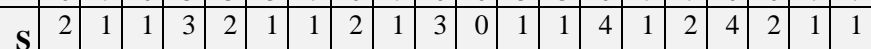

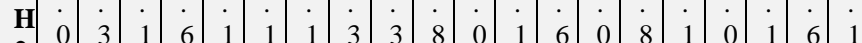
\begin{tabular}{l|llllllllllllllllllllll}
$\mathbf{2}$ & 0 & 3 & 1 & 6 & 1 & 1 & 1 & 3 & 3 & 8 & 0 & 1 & 6 & 0 & 8 & 1 & 0 & 1 & 6 & 1 \\
\hline & 0 & 3 & 7 & 7 & 7 & 7 & 7 & 3 & 3 & 3 & 0 & 7 & 7 & 0 & 3 & 7 & 0 & 7 & 7 & 7 \\
\hline
\end{tabular}

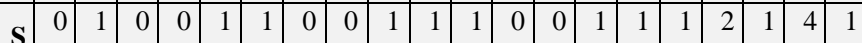

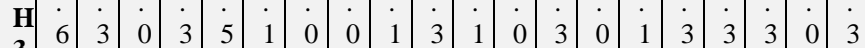
\begin{tabular}{llllllllllllllllllllllllll}
3 & 6 & 3 & 3 & 3 & 5 & 1 & 0 & 0 & 1 & 3 & 1 & 0 & 3 & 0 & 1 & 3 & 3 & 3 & 0 & 3 \\
& 7 & 3 & 0 & 3 & 0 & 7 & 0 & 0 & 7 & 3 & 7 & 0 & 3 & 0 & 7 & 3 & 3 & 3 & 0 & 3 \\
\hline
\end{tabular} $\begin{array}{lllllllllllllllllllllllll}\mathbf{S} & 2 & 1 & 1 & 1 & 0 & 1 & 2 & 0 & 0 & 0 & 0 & 1 & 0 & 2 & 0 & 2 & 3 & 2 & 2 & 3 \\ \end{array}$

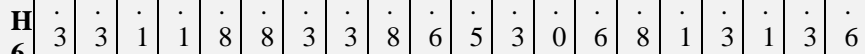
\begin{tabular}{lllllllllllllllllllllll}
$\mathbf{6}$ & 3 & 3 & 7 & 7 & 3 & 3 & 3 & 3 & 3 & 7 & 0 & 3 & 0 & 7 & 3 & 7 & 3 & 7 & 3 & 7 \\
\hline
\end{tabular} $\begin{array}{lllllllllllllllllllll}\mathbf{S} & 3 & 3 & 2 & 2 & 3 & 1 & 2 & 3 & 1 & 2 & 1 & 2 & 1 & 0 & 3 & 2 & 3 & 2 & 2 & 3\end{array}$ H

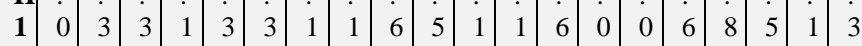
\begin{tabular}{llllllllllllllllllllllll}
$\mathbf{0}$ & 0 & 3 & 3 & 7 & 3 & 3 & 7 & 7 & 7 & 0 & 7 & 7 & 7 & 0 & 0 & 7 & 3 & 0 & 7 & 3 \\
\hline
\end{tabular} $\begin{array}{llllllllllllllllllllll}\mathbf{S} & 2 & 1 & 1 & 1 & 2 & 0 & 0 & 0 & 0 & 3 & 2 & 4 & 0 & 3 & 0 & 2 & 3 & 1 & 2 & 1\end{array}$ $\mathbf{H}$

$\begin{array}{lllllllllllllllllllll}1 & 5 & 8 & 3 & 3 & 0 & 0 & 3 & 1 & 3 & 3 & 1 & 0 & 6 & 6 & 0 & 3 & 8 & 1 & 3 & 1\end{array}$ \begin{tabular}{llllllllllllllllllllll}
1 & 0 & 3 & 3 & 3 & 0 & 0 & 3 & 7 & 3 & 3 & 7 & 0 & 7 & 7 & 0 & 3 & 3 & 7 & 3 & 7 \\
\hline
\end{tabular}

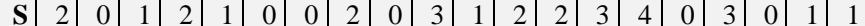
H

$\begin{array}{lllllllllllllllllllll}1 & 1 & 1 & 3 & 1 & 6 & 1 & 1 & 1 & 8 & 5 & 6 & 5 & 0 & 8 & 0 & 0 & 6 & 5 & 1 & 0\end{array}$ \begin{tabular}{lllllllllllllllllllllll}
3 & 7 & 7 & 3 & 7 & 7 & 7 & 7 & 7 & 3 & 0 & 7 & 0 & 0 & 3 & 0 & 0 & 7 & 0 & 7 & 0 \\
\hline
\end{tabular} $\mathbf{F}$ $\begin{array}{lllllllllllllllllllll}1 & 1 & 6 & 3 & 3 & 0 & 5 & 3 & 5 & 3 & 6 & 6 & 3 & 3 & 5 & 0 & 3 & 0 & 3 & 3 & 3\end{array}$ \begin{tabular}{llllllllllllllllllll}
7 & 7 & 3 & 3 & 0 & 0 & 3 & 0 & 3 & 7 & 7 & 3 & 3 & 0 & 0 & 3 & 0 & 3 & 3 & 3 \\
\hline
\end{tabular} F

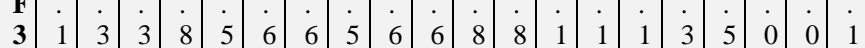
\begin{tabular}{lllllllllllllllllllll}
7 & 3 & 3 & 3 & 0 & 7 & 7 & 0 & 7 & 7 & 3 & 3 & 7 & 7 & 7 & 3 & 0 & 0 & 0 & 7 \\
\hline
\end{tabular} $\begin{array}{lllllllllllllllllllllll}1 & 2 & 1 & 2 & 1 & 2 & 0 & 0 & 3 & 1 & 0 & 0 & 1 & 1 & 1 & 0 & 1 & 0 & 0 & 1\end{array}$ $4 \begin{array}{llllllllllllllllllll}5 & 3 & 5 & 3 & 6 & 6 & 0 & 3 & 3 & 8 & 3 & 1 & 3 & 6 & 5 & 3 & 5 & 3 & 0 & 6\end{array}$ \begin{tabular}{llllllllllllllllllllll}
0 & 3 & 0 & 3 & 7 & 7 & 0 & 3 & 3 & 3 & 3 & 7 & 3 & 7 & 0 & 3 & 0 & 3 & 0 & 7 \\
\hline
\end{tabular}

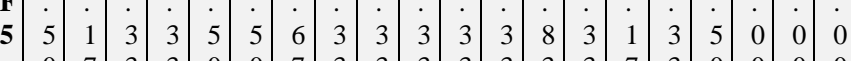
\begin{tabular}{lllllllllllllllllllll}
0 & 7 & 3 & 3 & 0 & 0 & 7 & 3 & 3 & 3 & 3 & 3 & 3 & 3 & 7 & 3 & 0 & 0 & 0 & 0 \\
\hline
\end{tabular}

Table: 5 Total relationship matrix for SBSC

\begin{tabular}{|c|c|c|c|c|c|c|c|c|c|c|c|c|c|c|c|c|c|c|c|c|}
\hline & $\mathbf{L}$ & $\mathbf{L}$ & $\mathbf{L}$ & $\mathbf{L}$ & $\mathbf{I}$ & $\mathbf{I}$ & $\mathbf{I}$ & $\mathbf{I}$ & $\mathbf{I}$ & $\mathbf{I}$ & $\mathbf{S}$ & $\mathbf{S}$ & $\mathbf{S}$ & $\mathbf{S}$ & $\mathbf{S}$ & $\mathbf{S}$ & & & & \\
$\mathbf{T}$ & $\mathbf{G}$ & $\mathbf{G}$ & $\mathbf{G}$ & $\mathbf{G}$ & $\mathbf{B}$ & $\mathbf{B}$ & $\mathbf{B}$ & $\mathbf{B}$ & $\mathbf{B}$ & $\mathbf{P}$ & $\mathbf{H}$ & $\mathbf{H}$ & $\mathbf{H}$ & $\mathbf{H}$ & $\mathbf{H}$ & $\mathbf{H}$ & $\mathbf{F}$ & $\mathbf{F}$ & $\mathbf{F}$ & $\mathbf{F}$ \\
& $\mathbf{2}$ & $\mathbf{3}$ & $\mathbf{4}$ & $\mathbf{5}$ & $\mathbf{P}$ & $\mathbf{P}$ & $\mathbf{P}$ & $\mathbf{P}$ & $\mathbf{P}$ & $\mathbf{7}$ & $\mathbf{2}$ & $\mathbf{3}$ & $\mathbf{6}$ & $\mathbf{1}$ & $\mathbf{1}$ & $\mathbf{1}$ & $\mathbf{1}$ & $\mathbf{3}$ & $\mathbf{4}$ & $\mathbf{5}$ \\
\hline $\mathbf{L}$ & 0 & 0 & 0 & 0 & 0 & 0 & 0 & 0 & 0 & 0 & 0 & 0 & 0 & 0 & 0 & 0 & 0 & 0 & 0 & 0 \\
$\mathbf{G}$ &. &. &. &. &. &. &. &. &. &. &. &. &. &. &. &. &. &. &. &. \\
$\mathbf{2}$ & 0 & 0 & 0 & 0 & 1 & 1 & 1 & 0 & 1 & 1 & 1 & 0 & 1 & 1 & 1 & 1 & 0 & 0 & 0 & 1 \\
& 7 & 7 & 7 & 8 & 0 & 1 & 0 & 7 & 0 & 3 & 0 & 7 & 1 & 5 & 0 & 0 & 9 & 7 & 9 & 3 \\
\hline $\mathbf{L}$ & 0 & 0 & 0 & 0 & 0 & 0 & 0 & 0 & 0 & 0 & 0 & 0 & 0 & 0 & 0 & 0 & 0 & 0 & 0 & 0 \\
$\mathbf{G}$ &. &. &. &. &. &. &. &. &. &. &. &. &. &. &. &. &. &. &. &. \\
$\mathbf{3}$ & 1 & 0 & 1 & 0 & 1 & 1 & 0 & 1 & 1 & 1 & 1 & 1 & 1 & 1 & 1 & 1 & 1 & 1 & 1 & 1 \\
& 2 & 7 & 0 & 9 & 3 & 2 & 7 & 1 & 3 & 6 & 4 & 2 & 0 & 8 & 3 & 3 & 7 & 1 & 1 & 3 \\
\hline $\mathbf{L}$ & 0 & 0 & 0 & 0 & 0 & 0 & 0 & 0 & 0 & 0 & 0 & 0 & 0 & 0 & 0 & 0 & 0 & 0 & 0 & 0 \\
$\mathbf{G}$ &. &. &. &. &. &. &. &. &. &. &. &. &. &. &. &. &. &. &. &. \\
$\mathbf{4}$ & 1 & 0 & 0 & 0 & 0 & 0 & 0 & 1 & 0 & 1 & 1 & 0 & 0 & 1 & 1 & 0 & 1 & 0 & 0 & 0 \\
& 0 & 5 & 4 & 9 & 9 & 7 & 4 & 0 & 7 & 3 & 2 & 5 & 6 & 4 & 0 & 9 & 4 & 7 & 8 & 8 \\
\hline $\mathbf{L}$ & 0 & 0 & 0 & 0 & 0 & 0 & 0 & 0 & 0 & 0 & 0 & 0 & 0 & 0 & 0 & 0 & 0 & 0 & 0 & 0 \\
$\mathbf{G}$ &. &. &. &. &. &. &. &. &. &. &. &. &. &. &. &. &. &. &. &. \\
$\mathbf{5}$ & 0 & 0 & 0 & 0 & 1 & 0 & 0 & 1 & 0 & 1 & 1 & 0 & 0 & 1 & 1 & 0 & 1 & 0 & 0 & 0 \\
\hline $\mathbf{I}$ & 8 & 5 & 8 & 5 & 2 & 7 & 4 & 1 & 8 & 4 & 3 & 5 & 4 & 5 & 0 & 8 & 4 & 8 & 9 & 8 \\
$\mathbf{B}$ & 0 & 0 & 0 & 0 & 0 & 0 & 0 & 0 & 0 & 0 & 0 & 0 & 0 & 0 & 0 & 0 & 0 & 0 & 0 & 0 \\
$\mathbf{P}$ & 1 & 0 & 0 &. &. &. &. &. &. &. &. &. &. &. &. &. &. &. &. &. \\
$\mathbf{1}$ & 0 & 9 & 6 & 7 & 7 & 0 & 0 & 0 & 1 & 1 & 1 & 0 & 0 & 1 & 0 & 0 & 0 & 0 & 1 & 0 \\
\hline
\end{tabular}

\begin{tabular}{|l|l|l|l|l|l|l|l|l|l|l|l|l|l|l|l|l|l|l|l|l|} 
I & 0 & 0 & 0 & 0 & 0 & 0 & 0 & 0 & 0 & 0 & 0 & 0 & 0 & 0 & 0 & 0 & 0 & 0 & 0 & 0
\end{tabular}

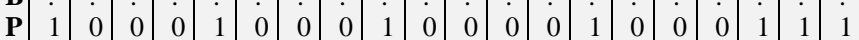
\begin{tabular}{llllllllllllllllllllll}
3 & 0 & 9 & 5 & 5 & 2 & 6 & 6 & 7 & 2 & 9 & 9 & 8 & 9 & 5 & 7 & 6 & 9 & 2 & 4 & 3 \\
\hline
\end{tabular} \begin{tabular}{|l|l|l|l|l|l|l|l|l|l|l|lllllllllllll|}
$\mathbf{I}$ & 0 & 0 & 0 & 0 & 0 & 0 & 0 & 0 & 0 & 0 & 0 & 0 & 0 & 0 & 0 & 0 & 0 & 0 & 0 & 0
\end{tabular} B

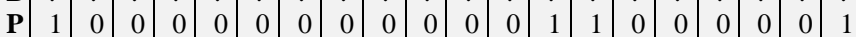
$\begin{array}{lllllllllllllllllllllllll}4 & 0 & 6 & 4 & 4 & 5 & 8 & 3 & 4 & 5 & 8 & 4 & 5 & 0 & 0 & 5 & 5 & 8 & 7 & 8 & 1\end{array}$

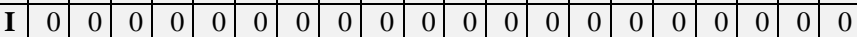
$\begin{array}{lllllllllllllllllllll}\mathbf{P} & 0 & 0 & 0 & 0 & 0 & 0 & 0 & 0 & 0 & 1 & 1 & 0 & 0 & 1 & 0 & 0 & 1 & 0 & 0 & 0\end{array}$ \begin{tabular}{lllllllllllllllllllllllll}
$\mathbf{5}$ & 8 & 5 & 8 & 7 & 9 & 7 & 4 & 5 & 6 & 3 & 2 & 5 & 5 & 4 & 9 & 9 & 3 & 7 & 8 & 8 \\
\hline
\end{tabular} $\begin{array}{llllllllllllllllllllllllll}\text { I } & 0 & 0 & 0 & 0 & 0 & 0 & 0 & 0 & 0 & 0 & 0 & 0 & 0 & 0 & 0 & 0 & 0 & 0 & 0 & 0\end{array}$

B

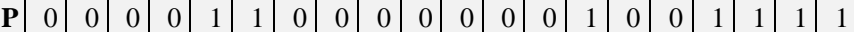
\begin{tabular}{lllllllllllllllllllllll}
6 & 9 & 9 & 4 & 5 & 2 & 2 & 3 & 5 & 6 & 9 & 8 & 7 & 4 & 4 & 8 & 6 & 2 & 2 & 3 & 1 \\
\hline
\end{tabular}

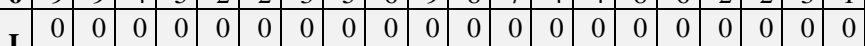
\begin{tabular}{lllllllllllllllll|l|l|l|l|l|l|l|}
$\mathbf{B}$ &. &. &. &. &. &. &. &. &. &. &. &. &. &. &. &. &. &. &. &. \\
7 & 1 & 0 & 0 & 1 & 1 & 0 & 0 & 1 & 1 & 1 & 1 & 1 & 0 & 1 & 1 & 1 & 1 & 0 & 1 & 0
\end{tabular} \begin{tabular}{lllllllllllllllllllll}
2 & 9 & 9 & 0 & 4 & 9 & 6 & 1 & 0 & 0 & 4 & 0 & 9 & 8 & 4 & 3 & 7 & 9 & 2 & 9 \\
\hline
\end{tabular} S $00 \begin{array}{lllllllllllllllllllllll} & 0 & 0 & 0 & 0 & 0 & 0 & 0 & 0 & 0 & 0 & 0 & 0 & 0 & 0 & 0 & 0 & 0 & 0 & 0\end{array}$

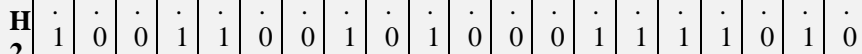
\begin{tabular}{|lllllllllllllllllllllllll}
2 & 0 & 8 & 7 & 1 & 1 & 8 & 5 & 0 & 8 & 4 & 7 & 7 & 7 & 6 & 0 & 0 & 5 & 9 & 0 & 8 \\
\hline
\end{tabular}

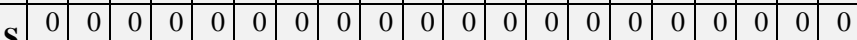

$\begin{array}{llllllllllllllllllllll}\mathbf{H} & \dot{0} & \dot{0} & \dot{0} & \dot{0} & \dot{0} & \dot{0} & 0 & 0 & 0 & 0 & 0 & 0 & 0 & 0 & 0 & 0 & 0 & 0 & 1 & 0\end{array}$ \begin{tabular}{lllllllllllllllllllllll}
4 & 5 & 2 & 3 & 6 & 5 & 2 & 3 & 6 & 6 & 5 & 3 & 3 & 7 & 5 & 5 & 8 & 5 & 1 & 6 \\
\hline
\end{tabular}

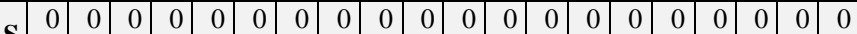

$\begin{array}{llllllllllllllllllllllll}\mathbf{H} & \dot{0} & \cdot & \cdot & \cdot & \cdot & \cdot & \cdot & \cdot & \cdot & \cdot & \cdot & \cdot & \cdot & \cdot & \cdot & \cdot & \cdot & \cdot & \cdot & \dot{0} \\ \mathbf{6} & 0 & 0 & 0 & 0 & 0 & 0 & 0 & 0 & 0 & 0 & 0 & 0 & 1 & 0 & 0 & 1 & 0 & 1 & 1\end{array}$ \begin{tabular}{lllllllllllllllllllllll}
9 & 7 & 5 & 6 & 7 & 8 & 7 & 5 & 6 & 7 & 6 & 6 & 4 & 2 & 6 & 8 & 2 & 8 & 0 & 1 \\
\hline
\end{tabular}

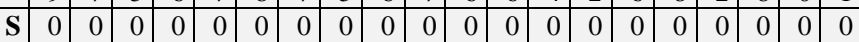
$\mathbf{H}$

$\begin{array}{lllllllllllllllllllllllllll}1 & 1 & 1 & 0 & 0 & 1 & 0 & 0 & 1 & 1 & 1 & 1 & 0 & 0 & 1 & 1 & 1 & 1 & 1 & 1 & 1\end{array}$ \begin{tabular}{lllllllllllllllllllllll}
$\mathbf{0}$ & 2 & 2 & 9 & 9 & 3 & 9 & 7 & 1 & 0 & 3 & 0 & 9 & 8 & 1 & 3 & 2 & 6 & 1 & 2 & 3 \\
\hline
\end{tabular}

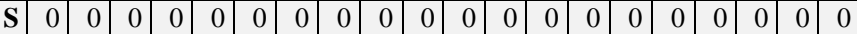
$\mathbf{H}$

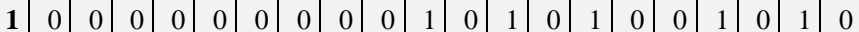
\begin{tabular}{llllllllllllllllllllllll}
1 & 9 & 8 & 6 & 7 & 9 & 5 & 3 & 5 & 6 & 2 & 9 & 1 & 5 & 4 & 6 & 9 & 4 & 7 & 0 & 7 \\
\hline
\end{tabular}

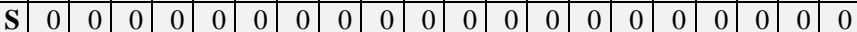
$\mathbf{H}$

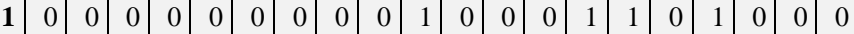
\begin{tabular}{lllllllllllllllllllll}
3 & 9 & 5 & 6 & 8 & 9 & 5 & 3 & 8 & 6 & 3 & 9 & 9 & 7 & 4 & 2 & 6 & 4 & 6 & 8 & 7 \\
\hline
\end{tabular} $\begin{array}{lllllllllllllllllllllllll}0 & 0 & 0 & 0 & 0 & 0 & 0 & 0 & 0 & 0 & 0 & 0 & 0 & 0 & 0 & 0 & 0 & 0 & 0 & 0\end{array}$

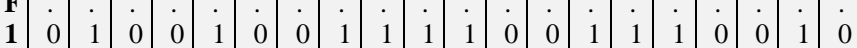
$\begin{array}{llllllllllllllllllllll}9 & 0 & 9 & 9 & 2 & 9 & 4 & 0 & 2 & 3 & 1 & 9 & 7 & 4 & 2 & 2 & 9 & 8 & 3 & 9\end{array}$

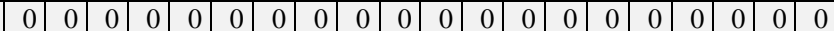
$\mathbf{F}$

$\begin{array}{llllllllllllllllllllll}3 & 0 & 0 & 0 & 0 & 0 & 0 & 0 & 0 & 0 & 0 & 0 & 0 & 0 & 0 & 0 & 0 & 0 & 0 & 1 & 0\end{array}$

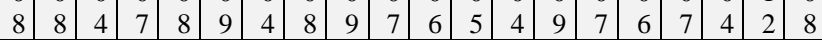

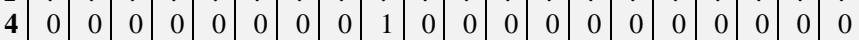
$\begin{array}{lllllllllllllllllllllll}7 & 8 & 5 & 7 & 8 & 9 & 2 & 4 & 0 & 8 & 5 & 4 & 5 & 9 & 7 & 5 & 8 & 5 & 5 & 7\end{array}$ $\begin{array}{lllllllllllllllllllllllll}0 & 0 & 0 & 0 & 0 & 0 & 0 & 0 & 0 & 0 & 0 & 0 & 0 & 0 & 0 & 0 & 0 & 0 & 0 & 0\end{array}$ $\begin{array}{lllllllllllllllllllllll}\mathbf{5} & 0 & 0 & 1 & 0 & 0 & 0 & 1 & 0 & 0 & 1 & 1 & 0 & 0 & 0 & 1 & 1 & 1 & 1 & 1 & 1 & 0\end{array}$ \begin{tabular}{|l|l|l|l|l|l|l|l|l|l|l|l|l|l|l|l|l|l|l|l|}
9 & 1 & 5 & 6 & 9 & 2 & 8 & 8 & 0 & 0 & 8 & 6 & 8 & 5 & 0 & 0 & 4 & 2 & 4 & 7 \\
\hline
\end{tabular}

\section{Results}

Based on the result of DEMATEL method, $(\mathrm{D}+\mathrm{R})$ value shows the importance of the whole system, while (D-R) value shows the effect of the factor on the system. If (D-R) value is positive then the factor is the cause factor, while if (D-R) is negative then the factor is effect factor $[31 ; 32]$. It is known that financial perspective is the most important perspective while the learning and growth and internal business process perspective are the cause factors and has an impact on the whole system. This is in accordance with the hierarchical structure of SBSC objectives that the learning and growth and internal business process will support the financial and stakeholder perspectives. 


\section{Conclusion}

In this study, "sustainability awards", "certification of environmental and social standards" and "resource productivity" are the three most important indicators with the greatest $(D+R)$ value. However, these three indicators are influenced by other indicators. To improve the sustainability of manufacturing companies, decision makers should focus on the cause-indicators group. Learning and growth and internal business process perspectives have positive (D-R) values which means these perspective are the causeindicators group. Therefore, improvisation in this perspective will improve corporate sustainability.

This study has contributed in providing decision makers with a systematic approach in establishing a visual strategy map with causal relationship among indicators. However, specific SBSC may differ from one company to another because each company in unique and has its own way of choosing the right indicators. However, this study has provided basic knowledge to establish a general SBSC strategy map in the manufacturing industry. Accordingly, the SBSC strategy map in this research can be useful reference for manufacturing companies.

\section{Acknowledgements}

The authors acknowledge the support of Universitas Indonesia through Hibah PITTA UI 2017

\section{References}

[1] Cherrafi, S. Elfezazi, A. Chiarini, and K. Benhida, "The integration of lean manufacturing, siz sigma and sustainability: A literature review and future research directions for developing a specific model," Journal of Cleaner Production, vo 139, pp. 828-246, 2016.

[2] W.P. Wong, and K.Y. Wong, "Synergizing an ecosphere of lean for sustainable operations, "Journal of Clean Production, vol 85, pp. 51-66, 2014.

[3] F. Figge, T. Hahn, S. Schaltegger, and M. Wagner, "The sustainability balanced scorecard - linking sustainabillity management to business strategy," Business Strategy and the Environment, vol 11, pp. 269-284, 2002.

[4] H.Y. Wu, "Constructing a strategy map for banking institutions with key performance indicators of the balanced scorecard," Evaluation and Program Planning, vol 35, pp. 303-320, 2012

[5] T. Supeekit, T. Somboonwiwat, and D Kritchanchai, "D MATELmodified ANP to evaluate internal hospital supply chain performance," Computers \& Industrial Engineering, vol 102, pp. 318-330, 2016.

[6] United Nations Industrial Developmen Organization (UNIDO), "The role of technology and innovation in inclusive and sustainable industrial development," Vienna, 2016.

[7] Deloitte, "Global Manufacturing Competitiveness Index, 2016.

[8] G. Aras, and D. Crowther, "Governance and sustainability," Management Decisions, vol 46, pp. 433-448, 2008.

[9] WCED (World Commission on Environment and Develoment), "Our common future," Oxford: Oxford University Press, 1987.

[10] F. Figge, T. Hahn, S. Schaltegger, and M. Wagner, "The sustainability balanced scorecard - linking sustainabillity management to business strategy," Business Strategy and the Environment, vol 11, pp. 269-284, 2002

[11] A. Lloret, "Modeling corporate sustainability strategy," Journal of Business Research, vol 69, pp. 418-425, 2016.

[12] F. Figge, T. Hahn, S. Schaltegger, and M. Wagner, "The sustainability balanced scorecard - linking sustainabillity management to business strategy," Business Strategy and the Environment, vol 11, pp. 269-284, 2002.

[13] T. Bieker, and B. Waxenberger, "Sustainability balanced scorecard and business ethics," Paper presenter at the greening of industry network conference, Goteborg, Sweden, 2002.

[14] R. Kaplan, and D. Norton, "The balanced scorecard: translating strategy into action," Harvard School Press, 1996

[15] R. D. Banker, H.M. Chang, J. Pizzini, "The balanced scorecard: judgmenal effects of performance measures linked to strategy," The Accounting Review, vol 79, pp. 1-23, 2004.
[16] N. Evans, "Assessing the balanced scorecard as a management tool for hotels," International Journal of Contemporary Hospital Management, vol 17, pp. 376-390, 2007.

[17] N. Makhijani, and J. Creelman, "How leading organizations successfully implement corporate strategy with the balanced scorecard," The OTI Thought Leadership Series, vol 1, pp. 1-16, 2008.

[18] G.H. Tzeng, C.H. Chiang, and C.W. Li, "Evaluating intertwined effects in e-learning programs: a novel hybrid MCDCM model based on factor analysis and DEMATEL," Expert Systems with Application, vol 32, pp. 1028-1044, 2007.

[19] M. Dytczak, and G. Ginda, "Is explicit processing of fuzzy direct influence evaluations in DEMATEL indispensable?" Expert Systems with Applications, vol 40, pp. 2027-2032.

[20] C.Y. Hsu, K.T. Chen, and G.H. Tzeng, "FMCDM with fuzzt DEMATEL approach for customers choice behavior model," International Journal Fuzzy System, vol 9, pp. 236-246, 2007.

[21] C.C. Hsu, Évaluation criteria for blog design and analysis of causal relationship using factor analysis and DEMATEL," Expert Systems with Application, vol 39, pp. 187-193, 2012.

[22] Hsu, A.H. Hu, C. Chiou, and T. Chen, "Using the FDM and ANP to construct a sustainability balanced scorecard for the semiconductor industry," Experts Systems with Applications, vol 38, pp. 1289112899,2011

[23] Indonesia Ministy of Industry, Pedoman Penilaian Penghargaan Industri Hijau, 2014.

[24] W.W. Wu, "Choosing knowledge management strategies by using a combined ANP and DEMATEL approach," Expert Systems with Application, vol 35, pp. 828-835, 2008

[25] C.L. Lin, and G.H. Tzeng, "A value-created system of science (technology) park by using DEMATEL," Expert Systems with Applications, vol 36, pp. 9683-9697, 2009.

[26] J.J.H. Liou, G.H. Tzeng, and H.C. Chang, "Airline safety measurement using a novel hybrid model." Journal of Air Transportation Management, vol 13, pp. 243-249, 2007.

[27] W.W. Wu, and Y.T. Lee, "Developing global managers competencies using the fuzzy DEMATEL method," Expert Systems with Applications, vol 32, pp. 499-507, 2007.

[28] Y.J. Chiu, H.C. Chen, G.H. Tzeng, and J.Z. Shyu, Marketing strategy based on customer behaviour for the LCD-TV," International Journal of Management Decision Making, vol 7, pp. 143-165, 2006.

[29] C.L. Lin, and G.H. Tzeng, "A value-created system of science (technology) park by using DEMATEL," Expert Systems with Applications, vol 36, pp. 9683-9697, 2009.

[30] T. Supeekit, T. Somboonwiwat, and D Kritchanchai, “ DEMATELmodified ANP to evaluate internal hospital supply chain performance," Computers \& Industrial Engineering, vol 102, pp. 318-330, 2016.

[31] Y.C. Lee, T.M. Yen, and C.H. Tsai, "Using importanceperformance analysis and decision making trial and evaluation laboratory to enhance order-winner criteria - A study of computer industry," Information Technology Journal, vol 7, pp. 396-408, 2008.

[32] G.H. Tzeng, C.H. Chiang, and C.W. Li, "Evaluating intertwined effects in e-learning programs: a novel hybrid MCDCM model based on factor analysis and DEMATEL," Expert Systems with Application, vol 32, pp. 1028-1044, 2007. 